\section{Giant cell arteritis, infections and biologics}

To the editor,

Numerous infectious agents have been implicated in the aetiology and/or pathogenesis of systemic vasculitides through direct damage of the vessel wall or autoimmune disorders. Mechanisms by which pathogens cause autoimmunity may include molecular mimicry (cross-reactivity between pathogenderived and self-derived epitopes), epitope spreading (the immune response to a persisting pathogen), bystander activation (non-specific activation of autoimmune cells by the inflammatory environment during infection) or immune response to cryptic antigens (subdominant epitopes which are normally hidden from T-cell recognition). ${ }^{1}$ The causative role of infectious agents is clearly established in polyarteritis nodosa and cryoglobulinemic vasculitis that are commonly associated with hepatitis $\mathrm{B}$ virus and hepatitis $\mathrm{C}$ virus (HCV), respectively. Microbial pathogens may probably contribute to the development of other systemic vasculitides as well. However, the evidence for a definitive link between infection and induced autoimmunity in many vasculitides is less strong or lacking. Epidemiological studies showing an increased incidence of systemic vasculitis in people infected with a particular agent, while not wholly definitive, may strengthen the infection-induced autoimmunity concept. ${ }^{1}$

In the impressive nested case-control study recently published in the Annals of the Rheumatic Diseases, ${ }^{2}$ Rhee et al examined the relationship between any infection or herpes zoster infection and the development of giant cell arteritis (GCA) in a large cohort of patients $(n=4559)$ and controls $(n=22759)$. Herpes zoster was associated with a higher risk of developing GCA $(p<0.01)$. However, the incidence rate ratio was relatively low (1.17) and suggests that clinically overt herpes zoster is unlikely to play a major causal role in the pathogenesis of GCA. These data contradict an earlier study that showed the presence of Varicella-zoster virus (VZV) antigen in the majority of GCA-positive temporal artery biopsies, supporting the hypothesis that VZV may trigger GCA. ${ }^{3}$ Rhee et al study does not refute a link between VZV and GCA, since the authors did not evaluate latent VZV infection or reactivation of virus that can explain histological findings in the temporal arteries in the absence of clinical signs and symptoms of infection. It is tempting to speculate that persistence of VZV during glucocorticoid therapy may account for the recurrent course of GCA in a proportion of patients. Therefore, proof-of-concept trial may be justified to study whether antiviral treatment with acyclovir or valacyclovir confers additional benefit to patients with GCA receiving corticosteroids. The excellent results of treatment with newer direct antiviral agents for $\mathrm{HCV}$-associated cryoglobulinemic vasculitis indirectly support feasibility of such approach. Notably, VZV is an established aetiological agent of central nervous system vasculitis (or vasculopathy) that can involve large and small intracranial vessels and is typically treated with intravenous acyclovir. ${ }^{4}$

In Rhee et al study, the risk of incident GCA was higher among patients who had any prior infections though this association was also quite modest (the incidence rate ratio of 1.26). However, it was 'dose dependent', and the incidence rate ratio reached 2.18 in patients with a history of $\geq 5$ infections. The authors suggested that infections may be directly involved in the pathogenesis of GCA or may be just a marker of immune dysfunction. In a previous matched historical cohort study, Durand and Thomas showed that treated patients with GCA were also at increased risk of infections, particularly in the first few months following diagnosis. ${ }^{6}$ In the latter study, an increase in the risk of lower respiratory tract and urinary infections during immunosuppressive treatment was comparable to that in Rhee et al study. In a smaller population-based retrospective cohort study, there was no overall increased risk of infections requiring or acquired during hospitalisation in patients with GCA who were taking glucocorticoid therapy. ${ }^{7}$ Therefore, patients with GCA may be more prone to infections than controls even prior to onset of systemic vasculitis, while glucocorticoid therapy does not seem to increase significantly the occurrence of infections in these patients.

In our opinion, these findings have important implications for a choice of treatment. Currently, we face an increase in the number of clinical trials evaluating different biological agents as a treatment for recurrent or newly diagnosed GCA. ${ }^{8}$ A list of promising biological agents that may be beneficial in patients with GCA already includes tocilizumab, abatacept, sirukumab and ustekinumab. It is expected that a wider use of biological agents will avoid multiple adverse events related to glucocorticoids. The steroid-sparing effect of biological therapy will be particularly valuable for the elderly patients with GCA who frequently develop metabolic complications during treatment with glucocorticoids. However, we should keep an eye on the risk of infections that can be associated with biological therapy. In the recent systematic review, Singh et $a l^{9}$ identified 106 clinical trials that reported serious infections and included patients with rheumatoid arthritis who received biological therapy. Compared with traditional disease-modifying antirheumatic drugs (DMARDs), standard-dose and high-dose biological agents were associated with an increased risk of serious infections, although low-dose biological agents were not. The absolute increase in the number of serious infections per 1000 patients treated each year ranged from 6 for standard-dose biological drugs to 55 for combination biological therapy, compared with traditional DMARDs. Biological agents have different mechanisms of action and safety profiles. Therefore, it would be a mistake to assume that all of them increase the risk of infections compared with standard treatment. However, the balance between possible benefit and harm should be carefully evaluated.

In summary, Rhee et al study suggests that clinically overt herpes zoster infections play a minor role, if any, in the pathogenesis of GCA. However, this case-control study does not rule out the hypothesis that latent or subclinical VZV infection may contribute to the development of GCA. Meanwhile, the clinical significance of VZV antigen identification in the temporal arteries should not be overstated. Numerous studies demonstrated associations between a large variety of pathogens and atherosclerosis, partly by the presence of the infectious agent (eg, Chlamydophila pneumoniae) in the human atherosclerotic tissue. ${ }^{10}$ Many molecular mechanisms have been suggested by which microbes may affect atherogenesis. Nevertheless, in the large-scale randomised clinical trials, evaluating the efficacy of antibiotic treatment for the secondary prevention of coronary events, there was no reduction in the rate of cardiovascular events, thereby challenging the validity of the infection hypothesis. ${ }^{11}$ The most compelling proof of infectious theory of GCA would be disappearance of symptoms or prevention of recurrences with the clearance of the infection. The story of infections and systemic vasculitides will be continued.

\section{Sergey Moiseev, ${ }^{1}$ Pavel Novikov, ${ }^{1}$ Ilya Smitienko, ${ }^{2}$ Elena Shchegoleva ${ }^{1}$}

${ }^{1}$ Clinic of Nephrology, Internal and Occupational Diseases, Sechenov First Moscow State Medical University, Moscow, Russia 
${ }^{2}$ Russian University of Peoples' Friendship, Moscow, Russia

Correspondence to Professor Sergey Moiseev, Clinic of Nephrology, Internal and Occupational Diseases, Sechenov First Moscow State Medical University, Rossolimo, 11/5, Moscow 119435, Russia;

clinpharm@mtu-net.ru

Contributors All authors contributed to review of the manuscript.

Competing interests None declared.

Provenance and peer review Not commissioned; internally peer reviewed.

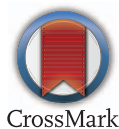

To cite Moiseev S, Novikov P, Smitienko I, et al. Ann Rheum Dis 2017;76:e29.

Received 11 December 2016

Accepted 14 December 2016

Published Online First 5 January 2017

\section{S Linked}

http://dx.doi.org/10.1136/annrheumdis-2016-210976

Ann Rheum Dis 2017;76:e29. doi:10.1136/annrheumdis-2016-210955

\section{REFERENCES}

1 Ercolini AM, Miller SD. The role of infections in autoimmune disease. Clin Exp Immunol 2009;155:1-15.
2 Rhee RL, Grayson PC, Merkel PA, et al. Infections and the risk of incident giant cell arteritis: a population-based, case-control study. Ann Rheum Dis 2017;76:1031-5.

3 Gilden D, White T, Khmeleva N, et al. Prevalence and distribution of VZV in temporal arteries of patients with giant cell arteritis. Neurology 2015;84:1948-55.

4 Gilden D, Cohrs RJ, Mahalingam R, et al. Varicella zoster virus vasculopathies: diverse clinical manifestations, laboratory features, pathogenesis, and treatment. Lancet Neurol 2009:8:731-40.

5 Nagel MA, Cohrs RJ, Mahalingam R, et al. The varicella zoster virus vasculopathies: clinical, CSF, imaging, and virologic features. Neurology 2008;70:853-60

6 Durand M, Thomas SL. Incidence of infections in patients with giant cell arteritis: a cohort study. Arthritis Care Res (Hoboken) 2012; 64:581-8.

7 Udayakumar PD, Chandran AK, Crowson CS, et al. Hospitalized infections in giant cell arteritis: a population-based retrospective cohort study. I Rheumatol 2014:41:2447-51.

8 Moiseev S, Novikov P, Meshkov A, et al. Biologic agents for giant cell arteritis: treat to target. Ann Rheum Dis 2016;75:e58.

9 Singh JA, Cameron C, Noorbaloochi S, et al. Risk of serious infection in biological treatment of patients with rheumatoid arthritis: a systematic review and meta-analysis. Lancet 2015;386:258-65.

10 Campbell LA, Rosenfeld ME. Infection and atherosclerosis development. Arch Med Res 2015;46:339-50.

11 Stassen FR, Vainas T, Bruggeman CA. Infection and atherosclerosis. An alternative view on an outdated hypothesis. Pharmacol Rep 2008;60:85-92. 